Research Article

\title{
Effects of Chengqi Decoction on Complications and Prognosis of Patients with Pneumonia-Derived Sepsis: Retrospective Cohort Study
}

\author{
Zhipeng Huang, ${ }^{1,2}$ Xiaoxin Cai, ${ }^{2}$ Yao Lin, ${ }^{2}$ Bojun Zheng $\left(\mathbb{D},{ }^{3}\right.$ Li Jian $\left(\mathbb{D},{ }^{3}\right.$ Yu Yi $\left(\mathbb{D},{ }^{3}\right.$ \\ and Yang Guang $\mathbb{1}^{3}$ \\ ${ }^{1}$ Dongguan Hospital of Integrated Chinese and Western Medicine Affiliated to Guangzhou \\ University of Traditional Chinese Medicine, Dongguan 523000, Guangdong, China \\ ${ }^{2}$ Guangzhou University of Traditional Chinese Medicine, Guangzhou 510405, Guangdong, China \\ ${ }^{3}$ Department of Critical Care Medicine, The Second Affiliated Hospital of Guangzhou University of Traditional Chinese Medicine, \\ Guangzhou 510006, Guangdong, China
}

Correspondence should be addressed to Li Jian; lijian426@gzucm.edu.cn, Yu Yi; yuyi27@mail2.sysu.edu.cn, and Yang Guang; yangguang@gzucm.edu.cn

Received 16 August 2021; Accepted 11 October 2021; Published 29 October 2021

Academic Editor: Zhaohui Liang

Copyright (c) 2021 Zhipeng Huang et al. This is an open access article distributed under the Creative Commons Attribution License, which permits unrestricted use, distribution, and reproduction in any medium, provided the original work is properly cited.

Purpose. A specific and efficacious method for treatment of pneumonia-derived sepsis is lacking. Chengqi decoction has been used for treatment of pneumonia-derived sepsis, but a clinical trial on patients with pneumonia-derived sepsis is lacking, a gap in the literature that we sought to fill. Patients and Methods. 282 patients with pneumonia-derived sepsis admitted to the intensive care unit of our hospital were selected. They were divided into the treatment group (141 cases) and control group (141 cases). Both groups underwent conventional treatment, but Chengqi decoction (in the form of enema) was given to the treatment group. Mortality, morbidity (abdominal distension and gastrointestinal bleeding), duration of antibiotic use, and use of vasoactive agents were documented 28 days after the drug was used. Results. The treatment group reduced mortality and morbidity (abdominal distension) $(P<0.05)$. After adjustment for significant covariates, 28-day survival was similar for the whole group (hazard ratio (HR): $0.48 ; 95 \%$ confidence interval $(\mathrm{CI}): 0.23-0.97 ; P=0.037)$, for the subgroup $(n=120)$ with Acute Physiology and Chronic Health Evaluation II score $\geq 25$ (HR: 0.180; 95\% CI: 0.032-0.332; $P=0.039)$ and for the subgroup $(n=66)$ with N-terminal B-type natriuretic peptide $<1800(0.059,0.004-0.979$, and 0.019$)$. There was no difference between the two groups for the duration of antibiotic use, major bleeding, or use of vasoactive drugs. Conclusions. Chengqi decoction improved 28-day survival and reduced the prevalence of abdominal distension in patients with pneumonia-derived sepsis.

\section{Introduction}

Sepsis is a clinical syndrome involving physiological, biological, and biochemical abnormalities and life-threatening organ dysfunction caused by a dysregulated inflammatory response to infection. Sepsis and septic shock are major healthcare problems [1]. Sepsis is considered a time-sensitive emergency because the best chance for a patient to survive is to be treated promptly [2]. However, the therapeutic effect of sepsis is not satisfactory, and the mortality rate is high [3].

Severe pneumonia is caused by infection by pathogenic microorganisms and is the main infection site of sepsis [4]. Unfortunately, patients suffering from severe sepsis often develop intestinal injury, which hampers treatment [5]. Therefore, exploration of any method that can cure both pneumonia and intestinal injury is rational and urgent. 
Traditional Chinese medicine (TCM) theory dictates that diseases of the lung and those of the large intestine react with each other. The occurrence and development of sepsis is related to translocation of bacterial/endotoxins in the intestines [6, 7]. However, enemas containing TCM formulations have important roles in the immunomodulation of sepsis [8].

"Chengqi decoction" (CD) is a well-known TCM formulation used commonly in China. CD has an anti-inflammatory role in sepsis and prevents translocation of intestinal flora. TCM theory also states "Fei he da chang"- "the lung and the large intestine being interiorexteriorly related" [9]. If intestinal failure occurs, a large volume of toxins accumulates in the intestinal tract, so using enemas containing TCM formulations could become a new method of sepsis treatment [10].

We wished to ascertain if $\mathrm{CD}$ can be used to reduce the risk of death from pneumonia-derived sepsis (PDS), abdominal distension, gastrointestinal bleeding, use of vasoactive drugs, and duration of antibiotic use. We also sought to identify the characteristics of CD that could reduce the risk of death from PDS by 28 days by relieving gastrointestinal complications.

\section{Patients and Materials}

2.1. Study Design. We conducted a retrospective, observational cohort study and reported its results in accordance with Strengthening the Reporting of Observational Studies in Epidemiology (STROBE) guidelines [11].

2.2. Study Setting and Population. This study was conducted in a 15-bed intensive care unit (ICU) at a university-affiliated tertiary-care hospital. Patients admitted to the ICU between March 2014 and September 2019 were evaluated for study inclusion. For all patients, the characteristics documented at baseline were age, sex, weight, height, body mass index (BMI), diagnosis upon hospital admission, Sequential Organ Failure Assessment (SOFA) score, Acute Physiology and Chronic Health Evaluation II (APACHE II) score, heart rate, systolic blood pressure, diastolic blood pressure, mean arterial pressure, central venous pressure, white blood cell count, neutrophilic granulocyte percentage, $\mathrm{pH}$, arterial partial pressure of oxygen $\left(\mathrm{PaO}_{2}\right)$, arterial partial pressure of carbon dioxide $\left(\mathrm{PaCO}_{2}\right), \mathrm{PaO}_{2} /$ fraction of inspired oxygen $\left(\mathrm{FiO}_{2}\right)$, central (mixed) venous oxygen saturation $\left(\mathrm{ScvO}_{2}\right)$, as well as levels of lactate, $\mathrm{N}$-terminal B-type natriuretic peptide (NT-proBNP), creatinine, blood urea nitrogen (BUN), C-reactive protein, and procalcitonin.

Patients (male or female) were aged $>18$ years. Patients were restricted to those with proven infections, defined as a positive blood culture or positive bronchoalveolar lavage fluid (BALF) culture and SOFA score $\geq 2$; within $24 \mathrm{~h}$ of time, the culture was ordered [12]. Cultures positive for common contaminants (e.g., coagulase-negative staphylococci in one of two blood culture bottles and Candida species in BALF cultures) were excluded.
The control group comprised sepsis patients who had been in the ICU $>7$ days. Patients were excluded from the control group if they had a malignant tumor and advanced cachexia, had a tendency for severe bleeding or coagulation disorder, were pregnant or lactating, were infection of other systems, such as the urinary system and digestive tract infection, and had contraindications such as acute abdomen and severe cardiovascular diseases.

2.3. Variables. Mortality at 28 days was documented. "Abdominal distension" was defined as abdominal pressure $>12 \mathrm{mmHg}$ [13]. "Gastrointestinal bleeding" was defined as occult blood in feces or succus gastricus after exclusion of hemorrhoids or oral bleeding. The duration of antibiotic treatment and use of vasoactive agents were also documented.

2.4. Treatment. Conventional treatment (e.g., infection prevention, organ function support, resuscitation after shock, correction of disturbances in water and electrolyte balance, and maintenance of acid-base balance) and a lungprotective ventilation strategy (if necessary) were conducted for patients in both groups. On that basis, enema therapy using CD was added in the treatment group.

Rheum officinale, mirabilite, lobster sauce, Fructus Aurantii Immaturus, and Magnolia officinalis were the basic prescriptions for $\mathrm{CD}$, with addition or subtraction of components as needed. Then, $200 \mathrm{~mL}$ of CD was fried strongly at $37^{\circ} \mathrm{C}$. Enema containing $\mathrm{CD}$ was given once a day over 7 days.

2.5. Statistical Analyses. Patients were monitored after enrollment to 28 days or until death. Baseline characteristics were assessed within $24 \mathrm{~h}$ before enrollment. Data were analyzed using SPSS 25.0 (IBM, Armonk, NY, USA). Differences between the treatment group and control group were tested by analysis of an unpaired $t$-test, Wilcoxon's rank-sum test, $x^{2}$ test, or Fisher's exact test, as appropriate. Values are the mean \pm SD. $P<0.05$ was considered significant.

Univariate Cox regression was used to model the odds of 28-day mortality. For categorical variables, HRs reflected the increased odds of 28-day mortality for absence of the variables. For continuous variables, HRs reflected the increased odds of 28-day mortality for a one-unit increase in the baseline variable. The meaningful factors of single-factor analysis were introduced into the Cox multiple regression model. Factor screening was based on gradual introduction of a removal method to calculate the HR. In addition, we undertook multivariate Cox regression analysis to model the odds of 28-day mortality using the APACHE II score, NT-proBNP level, age, lactate level, $\mathrm{PaO}_{2} / \mathrm{FiO}_{2}, \mathrm{ScvO}_{2}$, gastrointestinal bleeding, abdominal distension, and $\mathrm{BMI}$ as independent variables. A multivariate model using the APACHE II score, NT-proBNP level, and age was also used for analyses. Multivariate models reported HRs adjusted for all variables in the model. $P<0.05$ was considered significant for all comparisons. 
TABle 1: Patient characteristics at baseline.

\begin{tabular}{|c|c|c|c|c|}
\hline & Total & Chengqi decoction & Control & $P$ \\
\hline Patients $(n)$ & 282 & 141 & 141 & - \\
\hline Male $(n(\%))$ & $162(100)$ & $75(46.29)$ & $87(53.70)$ & 0.223 \\
\hline Age (years) & $67.00(67.00-87.25)$ & $67.00(48.00-87.00)$ & $67.00(55.00-90.00)$ & 0.484 \\
\hline BMI $\left(\mathrm{kg} / \mathrm{m}^{2}\right)$ & $23.66(21.25-25.39)$ & $24.22(22.04-30.48)$ & $23.31(20.29-25.39)$ & 0.006 \\
\hline APACHE II score & $24.30 \pm 7.20$ & $25.23 \pm 6.33$ & $23.40 \pm 8.00$ & 0.222 \\
\hline SOFA score & $12.00(11.00-15.00)$ & $13.00(11.00-15.00)$ & $12.00(10.00-14.00)$ & 0.189 \\
\hline HR (bpm) & $94.00(78.00-113.25)$ & $88.00(60.00-102.00)$ & $97.00(89.00-115.00)$ & 0.010 \\
\hline SBP (mmHg) & $115.00(105.00-127.50)$ & $115.00(105.00-126.00)$ & $111.00(98.00-129.00)$ & 0.803 \\
\hline DBP (mmHg) & $49.50(45.00-62.25)$ & $50.00(44.00-63.00)$ & $49.00(45.00-61.00)$ & 0.771 \\
\hline MAP (mmHg) & $72.00(63.00-85.50)$ & $72.00(62.00-83.00)$ & $72.00(65.00-88.00)$ & 0.688 \\
\hline CVP (mmHg) & $8.95 \pm 5.13$ & $8.62 \pm 5.52$ & $8.28 \pm 4.61$ & 0.204 \\
\hline WBC & $12.56(4.19-23.90)$ & $4.37(3.72-23.90)$ & $14.19(7.19-23.90)$ & 0.065 \\
\hline NG\% & $81.20(80.10-87.68)$ & $81.20(79.10-87.30)$ & $81.20(80.10-89.90)$ & 0.655 \\
\hline CRP (mg/L) & $73.00(45.75-85.00)$ & $73.00(34.00-89.00)$ & $74.00(54.00-84.00)$ & 0.303 \\
\hline Procalcitonin (ng/mL) & $5.11(2.50-12.30)$ & $3.12(1.60-7.30)$ & $8.10(4.50-23.90)$ & $\leq 0.001$ \\
\hline $\mathrm{PH}$ & $7.39(7.30-7.44)$ & $7.38(7.29-7.43)$ & $7.39(7.30-7.46)$ & 0.137 \\
\hline $\mathrm{PaO}_{2}$ & $102.00(91.00-126.00)$ & $102.00(91.00-126.00)$ & $108.00(90.40-126.00)$ & 0.955 \\
\hline $\mathrm{PaCO}_{2}$ & $46.90(44.00-60.40)$ & $45.80(41.50-59.10)$ & $46.90(44.50-61.00)$ & 0.101 \\
\hline $\mathrm{PaO}_{2} / \mathrm{FiO}_{2}$ & $232.77 \pm 64.61$ & $217.58 \pm 63.68$ & $247.95 \pm 62.14$ & 0.021 \\
\hline Lactate $(\mathrm{mmol} / \mathrm{L})$ & $1.95(1.30-3.30)$ & $1.79(1.30-2.60)$ & $2.10(1.30-4.00)$ & 0.246 \\
\hline $\mathrm{ScvO}_{2}$ & $64.31 \pm 13.81$ & $59.86 \pm 13.79$ & $68.69 \pm 12.35$ & 0.002 \\
\hline NT-proBNP (pg/mL) & $5348.50(2201.00-12180.00)$ & $2392.00(1569.00-7810.00)$ & $7899.00(3883.00-15015.00)$ & $\leq 0.001$ \\
\hline $\mathrm{SCr}(\mu \mathrm{mol} / \mathrm{L})$ & $184.39(97.16-280.80)$ & $113.39(95.34-280.00)$ & $188.00(154.90-308.00)$ & 0.106 \\
\hline $\mathrm{BUN}(\mathrm{mmol} / \mathrm{L})$ & $13.19(8.80-16.41)$ & $10.50(7.58-15.20)$ & $15.61(12.00-19.66)$ & $\leq 0.001$ \\
\hline
\end{tabular}

SOFA, sequential organ failure assessment; BMI, body mass index; APACHE, Acute Physiology and Chronic Health Evaluation; BUN, blood urea nitrogen; HR, heart rate; SBP, systolic blood pressure; DBP, diastolic blood pressure; MAP, mean arterial blood pressure; CVP, central venous pressure; WBC, white blood cell; $\mathrm{NG} \%$, neutrophilic granulocyte percentage; CRP, C-reactive protein; $\mathrm{PaO}_{2}$, arterial partial pressure of oxygen; $\mathrm{PaCO}_{2}$, arterial partial pressure of carbon dioxide; $\mathrm{PaO}_{2} / \mathrm{FiO}_{2}$, arterial partial pressure of oxygen/inspired oxygen fraction; $\mathrm{ScvO}_{2}$, central (mixed) venous oxygen saturation; NT-proBNP, Nterminal B-type natriuretic peptide; SCr, serum creatinine.

TABle 2: Primary and secondary outcomes.

\begin{tabular}{lccr}
\hline & Total & Chengqi decoction & Control \\
\hline Primary outcome & & & \\
$\quad$ Mortality $(n(\%))$ & $96(100)$ & $33(34.37)$ & $63(65.63)$ \\
\hline Secondary outcomes & & & \\
$\quad$ Abdominal distension $(n(\%))$ & $165(100)$ & $27(56.36)$ & $105(63.64)$ \\
Gastrointestinal bleeding $(n(\%))$ & $48(100)$ & $13.23 \pm 5.32$ & $21(43.75)$ \\
Duration of antibiotic use (days) & $12.92 \pm 5.31$ & $84(46.67)$ & $12.6 \pm 5.23$ \\
Vasoactive agent $(n(\%))$ & & & 0.02 \\
\hline
\end{tabular}

\section{Results}

141 patients were enrolled in the CD group, and other 141 pneumonia-suffering patients were enrolled as controls. The characteristics of the two groups of patients at baseline are given in Table 1. The groups were well-matched, except for BMI $(P<0.01)$, heart rate $(P=0.01)$, procalcitonin level $(P<0.01), \mathrm{FiO}_{2} / \mathrm{PaO}_{2}(P=0.02), \mathrm{ScvO}_{2} \%(P<0.01)$, NTproBNP level $(P<0.01)$, and BUN level $(P<0.01)$ in the treatment group versus the control group.

Data for primary and secondary outcomes are given in Table 2. By day 28, the treatment group had a significant reduction in mortality $(34.37 \%$ vs. $65.63 \% ; P=0.03)$ and prevalence of abdominal distension (36.36\% vs. $63.64 \%$; $P=0.02)$, as well as a nonsignificant increase in the prevalence of gastrointestinal bleeding (56.25\% vs. $43.75 \%$; $P=0.58)$. However, there was a nonsignificant reduction in use of vasoactive agents ( $46.67 \%$ vs. $53.53 \% ; P=0.39)$ and a nonsignificant decrease in duration of antibiotic use $(13.23 \pm 5.32$ vs. $12.6 \pm 5.23 ; P=0.33)$.

The number of cases in our study was relatively small, so the survival rate at a given time could not be calculated, and Kaplan-Meier analysis was used. There was a significant difference in survival at 28 days between the two groups (Figure 1). Survival of the treatment group was significantly higher than that in the control group. The log-rank test was also carried out and also revealed a significant difference between the two groups $(P=0.04)$.

We studied the sum populations to determine the risk factors for death in each group. We applied univariate analysis of 10 potential risk factors with mortality as the dependent variable to each group (Figure 2), and the results are given in Table 3 . We chose to include groups, the APACHE II score, NT-proBNP level, and age in the 


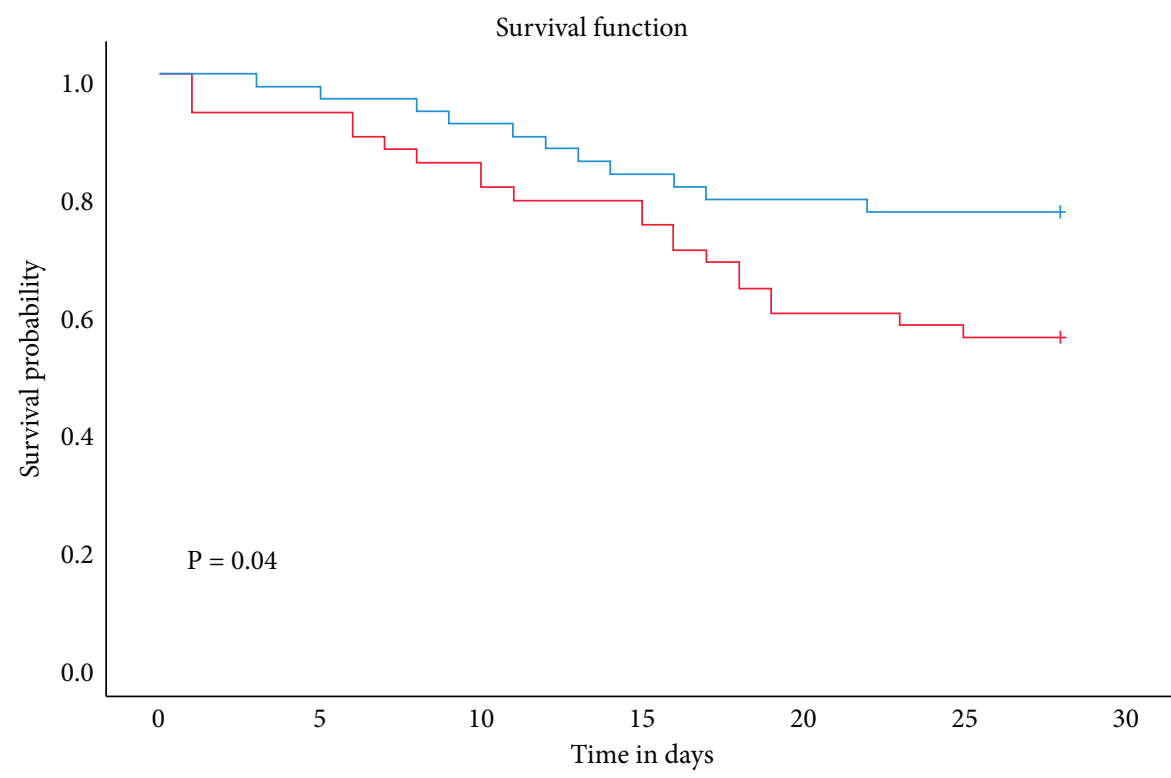

$\begin{array}{ll}\text { Group } & \\ \neg \text { Chengqi decoction } & + \text { Chengqi decoction-censor } \\ \neg \text { Control } & + \text { Control-censor }\end{array}$

Figure 1: Kaplan-Meier estimate of survival on day 28 after adjustment by Cox regression analysis for the NT-proBNP level, age, and APACHE II score. The red line corresponds to the control group. The blue line corresponds to the Chengqi decoction group. The HR for probability of survival at 28 days was 0.48 (95\% CI: $0.23-0.97 ; P=0.037$ ).

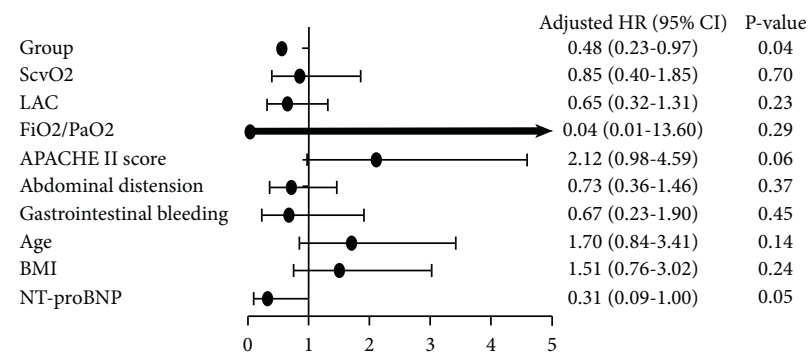

FIGURE 2: Univariate analysis of 10 potential risk factors for mortality as the dependent variable to each group.

TABLE 3: Univariate and multivariate models for overall survival on day 28.

\begin{tabular}{|c|c|c|c|}
\hline Covariate at study entry & HR & 95\% CI & $P$ \\
\hline \multicolumn{4}{|l|}{ Univariate models } \\
\hline NT-proBNP (pg/mL) (<1800 vs. $\geq 1800)$ & 0.31 & $0.09-1.00$ & 0.05 \\
\hline $\operatorname{BMI}\left(\mathrm{kg} / \mathrm{m}^{2}\right)(18-23$ vs. $>23$ or $<18)$ & 1.51 & $0.76-3.02$ & 0.24 \\
\hline Age (years) $(\leq 65$ vs. $>65)$ & 1.70 & $0.84-3.41$ & 0.14 \\
\hline Gastrointestinal bleeding (present vs. absent) & 0.67 & $0.23-1.90$ & 0.44 \\
\hline Abdominal distension (present vs. absent) & 0.73 & $0.36-1.46$ & 0.37 \\
\hline APACHE II score (<25 vs. $\geq 25)$ & 2.12 & $0.98-4.59$ & 0.06 \\
\hline $\mathrm{FiO}_{2} / \mathrm{PaO}_{2}(<150$ vs. $\geq 150)$ & 0.04 & $0.01-13.60$ & 0.29 \\
\hline Lactate $(\mathrm{mmol} / \mathrm{L})(<2$ vs. $\geq 2)$ & 0.65 & $0.32-1.31$ & 0.23 \\
\hline $\mathrm{ScvO}_{2}(\%)(<75$ vs. $\geq 75)$ & 0.85 & $0.40-1.85$ & 0.69 \\
\hline Group (Chengqi decoction vs. control) & 0.48 & $0.23-0.97$ & 0.04 \\
\hline \multicolumn{4}{|l|}{ Multivariate models } \\
\hline Group (Chengqi decoction vs. control) & 0.48 & $0.23-0.97$ & $0.04^{*}$ \\
\hline APACHE II score $(<25$ vs. $\geq 25)$ & 2.02 & $0.89-4.60$ & 0.09 \\
\hline NT-proBNP (pg/mL) (<1800 vs. $\geq 1800)$ & 0.34 & $0.09-1.19$ & 0.09 \\
\hline Age ( $\leq 65$ years vs. $>65$ years $)$ & 1.39 & $0.67-2.88$ & 0.38 \\
\hline
\end{tabular}

${ }^{*}$ Adjusted for: NT-proBNP, age, APACHE II score. HR, hazard ratio; NT-proBNP, N-terminal B-type natriuretic peptide; $\mathrm{BMI}_{\text {, body mass index; FiO }} / \mathrm{PaO}_{2}$, inspired oxygen fraction/arterial partial pressure of oxygen; $\mathrm{ScvO}_{2}$, central venous oxygen saturation; APACHE, Acute Physiology and Chronic Health Evaluation. 


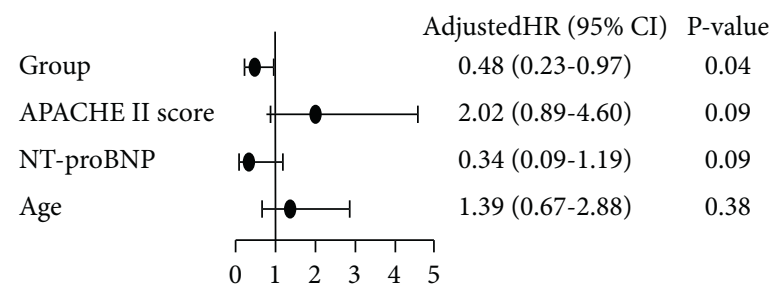

FIGURE 3: Multivariate analysis of four potential risk factors for mortality as the dependent variable to each group.

TABLE 4: Subgroup analyses of the risk of death in the Chengqi decoction group and control group at 28 days.

\begin{tabular}{|c|c|c|c|c|}
\hline Subgroup & Chengqi decoction $(n=141)$ & Control $(n=141)$ & HR with Chengqi decoction $(95 \% \mathrm{CI})$ & $P$ \\
\hline \multicolumn{5}{|l|}{ Age } \\
\hline$<65$ years, $n /$ total $(\%)$ & $18 / 63$ & $36 / 63$ & $0.30(0.08-1.08)$ & 0.06 \\
\hline$\geq 65$ years, $n /$ total $(\%)$ & $15 / 78$ & $27 / 78$ & $0.45(0.13-1.70)$ & 0.21 \\
\hline \multicolumn{5}{|l|}{ APACHE II score } \\
\hline$<25, n /$ total $(\%)$ & $27 / 78$ & $42 / 84$ & $0.53(0.18-1.58)$ & 0.25 \\
\hline$\geq 25, n /$ total $(\%)$ & $6 / 63$ & $21 / 57$ & $0.18(0.03-1.02)$ & 0.04 \\
\hline \multicolumn{5}{|l|}{ Lactate $(\mathrm{mmol} / \mathrm{L})$} \\
\hline$<2, n /$ total $(\%)$ & $15 / 81$ & $24 / 60$ & $0.34(0.09-1.28)$ & 0.10 \\
\hline$\geq 2, n /$ total $(\%)$ & $18 / 60$ & $39 / 81$ & $0.46(0.14-1.56)$ & 0.21 \\
\hline \multicolumn{5}{|l|}{ NT-proBNP (pg/mL) } \\
\hline$<1800, n /$ total $(\%)$ & $3 / 54$ & $6 / 12$ & $0.06(0.01-0.98)$ & 0.02 \\
\hline$\geq 1800, n /$ total $(\%)$ & $30 / 87$ & $57 / 129$ & $0.67(0.25-1.76)$ & 0.41 \\
\hline
\end{tabular}

HR, hazard ratio; APACHE, Acute Physiology and Chronic Health Evaluation.

multivariate model (Figure 3). After adjustment by univariable analysis, one covariate was found to be significant in the multivariate analysis, and the CD group showed a HR of 0.48 (95\% confidence interval (CI): $0.23-0.97 ; P=0.04$ ).

Subgroup analyses for the HR of death at days 28 are given in Table 4. In the subgroup with an APACHE II score $\geq 25$, the HR for probability of survival at 28 days was 0.18 (95\% CI: $0.03-1.02 ; P=0.04$ ). In the subgroup with NTproBNP $<1800 \mathrm{npg} / \mathrm{mL}$, the HR for the probability of survival at 28 days was 0.06 (95\% CI: $0.01-0.98 ; P=0.02$ ).

\section{Discussion}

In recent years, understanding of sepsis and septic shock has gone from the tissue level to cellular and molecular levels, that is, from the theory of microcirculatory ischemia and hypoxia to the current theory of excessive release of inflammatory factors. Some scholars believe that the displacement of toxins is due to severe trauma, infection, shock, sepsis, and septic shock after surgery [14]. The pathogenesis and treatment of sepsis have been studied deeply $[15,16]$, but the incidence of sepsis and septic shock has not improved considerably. PDS remains a challenge in extracorporeal circuits for drug delivery in critically ill patients, and the mortality in this patient population is high [17]. However, TCM has obvious advantages over Western medicine in PDS treatment and conforms to the theory of treating pulmonary diseases through intestinal administration of drugs. Giving sepsis patients enema containing the $\mathrm{CD}$ formulation, combined with conventional treatment, could elicit the advantages of TCM and Western medicine $[18,19]$.

This was the first retrospective cohort trial investigating the effects of a 7-day course of CD treatment in patients with PDS. In this study, we chose to include groups, the APACHE II score, NT-proBNP level, and age which were filtered through single-factor analysis in the multivariate model. Meanwhile, we found that groups was the most important influencing factor, so we further analyzed the 28-day mortality between the CD group and control group. CD treatment relieved abdominal distension and led to a significant decrease in 28-day mortality. CD treatment did not increase the prevalence of gastrointestinal bleeding, duration of antibiotic use, or use of vasoactive agents. There was a significant decrease in 28-day mortality between the two groups, especially in the subgroup with an APACHE II score $\geq 25$ and NT-proBNP $<1800 \mathrm{pg} / \mathrm{mL}$.

We demonstrated, through a retrospective cohort study, that CD could reduce 28-day mortality in patients with PDS. Improvement in the prognosis of patients with sepsis by $\mathrm{CD}$ has been demonstrated by several scholars [20-23]. In 2016, Mao et al. published a study on the effects of Xuan Bai Chengqi decoction (XCD) on lung compliance for patients with acute respiratory distress syndrome (ARDS). In that study, CD not only improved static compliance and dynamic compliance but also shortened the duration of parenteral nutrition and reduced the prevalence of complications and death [20]. CD also has a significant curative effect in severe pancreatitis, acute cholangitis, and myocardial ischemia [21-23]. 
Subgroup analyses revealed that patients with an APACHE II score $\geq 25$ and NT-proBNP $<1800 \mathrm{pg} / \mathrm{mL}$ were pronounced. These observations indicated that CD improved the prognosis of patients with PDS more significantly in the group with severe illness. In patients with good cardiac function, the effect of CD on the prognosis of patients with pneumonia and sepsis was more obvious and may have been associated with the increase of additional fluid in Chengqi decoction enema [24].

We found that CD could reduce the abdominal pressure of patients. This may have been related to the therapeutic characteristics of TCM formulations, which have multiple pathways, targets, and links. For example, Dachengqi decoction has been shown to be efficacious in ARDS treatment. Scholars have shown significant differences in the recovery time of intestinal sounds, anal exhaust time, regression of abdominal distension, as well as improvement in MODS and recovery time between two groups, indicating that Dachengqi decoction could improve the organ function of patients suffering from multiple-organ dysfunction syndrome [25-27]. Some scholars believe that TCM formulations have a good therapeutic effect on PDS and that they may act through the gastrointestinal tract. They have postulated that rhubarb can inhibit the activity of nitric oxide and inducible nitric oxide synthase to inhibit granulocyte aggregation and reduce free-radical production $[28,29]$. Our study showed that CD may improve PDS through reduction of intraabdominal pressure without increasing the risk of gastrointestinal bleeding. The reduction in intraabdominal pressure may have been due to a reduction in the intestinal inflammatory response by $\mathrm{CD}$.

Our study had three main limitations. First, it was conducted at a single institution. Second, the study cohort was small. Last, there was a potential selection bias to this retrospective study. Therefore, more prospective studies with larger cohorts are needed to support our findings.

\section{Conclusion}

In PDS, early administration of an enema containing CD for 7 days was safe and associated with improved survival without a significant increase in the risk of hemorrhage within the gastrointestinal tract. Our study was underpowered for an exploratory analysis to demonstrate survival benefit in patients with severe illness and good cardiac function.

\section{Data Availability}

The data used to support the findings of this study are included within the article.

\section{Disclosure}

Zhipeng Huang, Xiaoxin Cai, Yao Lin, and Bojun Zheng are the co-first authors..

\section{Conflicts of Interest}

The authors declare that they have no conflicts of interest.

\section{Authors' Contributions}

Bojun Zheng and Yu Yi designed the study. Zhipeng Huang, Xiaoxin Cai, and Yao Lin undertook the research and collected the data. Li Jian and Yang Guang analyzed the data. Li Jian wrote the manuscript. All authors contributed towards data analyses, drafting and revising the manuscript, and are accountable for all aspects of the work.

\section{Acknowledgments}

This work was supported by the Special Science and Technology Research of Guangdong Provincial Hospital of Traditional Chinese Medicine (YN 2014 PJR 203), the Guangdong Provincial Bureau of Traditional Chinese Medicine (20201157), the Guangdong Provincial Key Laboratory of Research on Emergency in TCM (2017B030314176), and the Science and Technology Planning Project of Guangdong Province of China (A2021100).

\section{References}

[1] M. Singer, C. S. Deutschman, C. W. Seymour, M. ShankarHari, D. Annane, and M. Bauer, "The third international consensus definitions for sepsis and septic shock (Sepsis-3)," Journal of the American Medical Association, vol. 315, no. 8, pp. 801-810, 2016.

[2] E. Abraham, "New definitions for sepsis and septic shock: continuing evolution but with much still to be done," Journal of the American Medical Association, vol. 315, no. 8, pp. 757-759, 2016.

[3] M. Cecconi, L. Evans, M. Levy, and A. Rhodes, "Sepsis and septic shock," Lancet, vol. 392, no. 10141, pp. 75-87, 2018.

[4] H. M. Ma, M. Ip, J. Woo et al., "Risk factors for drug-resistant bacterial pneumonia in older patients hospitalized with pneumonia in a Chinese population," An International Journal of Medicine, vol. 106, no. 9, pp. 823-829, 2013.

[5] S. A. Myers, L. Gobejishvili, S. Saraswat Ohri et al., "Following spinal cord injury, PDE4B drives an acute, local inflammatory response and a chronic, systemic response exacerbated by gut dysbiosis and endotoxemia," Neurobiology of Disease, vol. 124, pp. 353-363, 2019.

[6] B. H. Singer, R. P. Dickson, S. J. Denstaedt et al., "Bacterial dissemination to the brain in sepsis," American Journal of Respiratory and Critical Care Medicine, vol. 197, no. 6, pp. 747-756, 2018.

[7] B. W. Haak and W. J. Wiersinga, "The role of the gut microbiota in sepsis," Lancet Gastroenterol Hepatol, vol. 2, no. 2, pp. 135-143, 2017.

[8] T. B. Ahmad, L. Liu, M. Kotiw, and K. Benkendorff, "Review of anti-inflammatory, immune-modulatory and wound healing properties of molluscs," Journal of Ethnopharmacology, vol. 210, pp. 156-178, 2018.

[9] Q. Zhou and G. N. Verne, "Intestinal hyperpermeability: a gateway to multi-organ failure?" Journal of Clinical Investigation, vol. 128, no. 11, pp. 4764-4766, 2018.

[10] C. Smids, H. Talabur Horje, J. Drylewicz et al., "Intestinal $\mathrm{T}$ cell profiling in inflammatory bowel disease: linking $\mathrm{T}$ cell subsets to disease activity and disease course," Journal of Crohn's and Colitis, vol. 12, no. 4, pp. 465-475, 2018.

[11] E. Von Elm, D. G. Altman, M. Egger, S. J. Pocock, P. C. Gotzsche, and J. P. Vandenbroucke, "The strengthening the reporting of observational studies in epidemiology 
(STROBE) statement: guidelines for reporting observational studies," Annals of Internal Medicine, vol. 147, no. 8, pp. 573-577, 2007.

[12] E. P. Raith, A. A. Udy, M. Bailey et al., "Prognostic accuracy of the SOFA score, SIRS criteria, and qSOFA score for inhospital mortality among adults with suspected infection admitted to the intensive care unit," Journal of the American Medical Association, vol. 317, no. 3, pp. 290-300, 2017.

[13] M. L. N. G. Malbrain and I. E. De laet, "Intra-abdominal hypertension: evolving concepts," Critical Care Nursing Clinics of North America, vol. 24, no. 2, pp. 275-309, 2012.

[14] T. Van Der Poll, F. L. van de Veerdonk, B. P. Scicluna, and M. G. Netea, "The immunopathology of sepsis and potential therapeutic targets," Nature Reviews Immunology, vol. 17, no. 7, pp. 407-420, 2017.

[15] Y. Kakihana, O. Nishida, T. Taniguchi et al., "Efficacy and safety of landiolol, an ultra-short-acting beta 1-selective antagonist, for treatment of sepsis-related tachyarrhythmia (JLand 3S): a multicentre, open-label, randomised controlled trial," The Lancet Respiratory Medicine, vol. 8, no. 9, pp. 863-872, 2020.

[16] K. E. Rudd, S. C. Johnson, K. M. Agesa et al., "Global, regional, and national sepsis incidence and mortality, 1990-2017: analysis for the global burden of disease study," Lancet, vol. 395, no. 10219, pp. 200-211, 2020.

[17] M. Hites, A. M. Dell'Anna, S. Scolletta, and F. S. Taccone, "The challenges of multiple organ dysfunction syndrome and extracorporeal circuits for drug delivery in critically ill patients," Advanced Drug Delivery Reviews, vol. 77, pp. 12-21, 2014.

[18] R. Zeng, Y. Zheng, R. Fan et al., "Si-ni-tang (a Chinese herbal formula) for improving immunofunction in sepsis: study protocol for a pilot randomized controlled trial," Trials, vol. 20, no. 1, p. 537, 2019.

[19] G. Z. Zhao, R. B. Chen, B. Li et al., "Clinical practice guideline on traditional Chinese medicine therapy alone or combined with antibiotics for sepsis," Annals of Translational Medicine, vol. 7 , no. 6, p. 122, 2019.

[20] Z. Mao and H. Wang, "Effects of Xuanbai Chengqi decoction on lung compliance for patients with exogenous pulmonary acute respiratory distress syndrome," Drug Design, Development and Therapy, vol. 10, pp. 793-798, 2016.

[21] J. Guo, T. Jin, Z. Q. Lin et al., "Effect of Chaiqin Chengqi Decoction on cholecystokinin receptor 1-mediated signal transduction of pancreatic acinar cells in acute necrotizing pancreatitis rats," Chinese Journal of Integrative Medicine, vol. 21, no. 1, pp. 29-35, 2015.

[22] J. Guo, P. Xue, X. N. Yang et al., "The effect of Chaiqin Chengqi Decoction () on modulating serum matrix metalloproteinase 9 in patients with severe acute pancreatitis," Chinese Journal of Integrative Medicine, vol. 19, no. 12, pp. 913-917, 2013.

[23] L. Wang, Y. Li, Q. Ma et al., "Chaiqin Chengqi Decoction decreases IL-6 levels in patients with acute pancreatitis," Journal of Zhejiang University-Science B, vol. 12, no. 12, pp. 1034-1040, 2011.

[24] A. Kuriyama and S. Urushidani, "Continuous versus intermittent administration of furosemide in acute decompensated heart failure: a systematic review and meta-analysis," Heart Failure Reviews, vol. 24, no. 1, pp. 31-39, 2019.

[25] L. Y. Pan, Y. F. Chen, H. C. Li et al., "Dachengqi decoction attenuates intestinal vascular endothelial injury in severe acute pancreatitis in vitro and in vivo," Cellular Physiology and Biochemistry: International Journal of Experimental
Cellular Physiology, Biochemistry, and Pharmacology, vol. 44, no. 6, pp. 2395-2406, 2017.

[26] M. Z. Xie, Q. H. Qi, S. L. Zhang, and M. M. Wei, "Effects of Dachengqi decoction () on morphological changes in enteric nerve system of rats with multiple organ dysfunction syndrome," Chinese Journal of Integrative Medicine, vol. 21, no. 8, pp. 624-629, 2015.

[27] J. Yang, M. Xiao, X. Wang et al., "Dachengqi decoction reduces the serum levels of mast cell tryptase and inflammatory cytokines in rabbits with post-cardiac arrest syndrome," $X i$ Bao Yu Fen Zi Mian Yi Xue Za Zhi, vol. 30, no. 10, pp. 1026-1029, 2014.

[28] S. L. Yang, Y. Jin, and L. Shen, "Effects of dachengqi decoction containing serum on the expressions of caveolin-1, eNOS, and NF-kappaB in lipopolysaccharide stimulated human bronchial epithelial cells," Zhongguo Zhong Xi Yi Jie He Za Zhi, vol. 32, no. 8, pp. 1088-1094, 2012.

[29] X. G. Sun, Q. Fan, and Q. R. Wang, "Effect of dachengqi decoction on expressions of TLR4 and TNF-alpha in the lung and the large intestine of mice with endotoxemia," Zhongguo Zhong Xi Yi Jie He Za Zhi, vol. 31, no. 2, pp. 244-248, 2011. 